\title{
CORRECTION
}

\section{Correction: Factors associated with successful antipsychotic dose reduction in schizophrenia: a systematic review of prospective clinical trials and meta-analysis of randomized controlled trials}

Hideaki Tani, Shotaro Takasu, Hiroyuki Uchida (D), Takefumi Suzuki (D), Masaru Mimura and Hiroyoshi Takeuchi (DD

Neuropsychopharmacology (2020) 45:902; https://doi.org/10.1038/s41386-019-0598-y

Correction to: Neuropsychopharmacology https://doi.org/10.1038/ s41386-019-0573-7, published online 26 November 2019
In the original version of this Article, the DOI doi.org/10.1016/j. schres.2019.09.013 was incorrectly listed for reference 9. It should instead be listed for reference 10 . This has been corrected in both the PDF and HTML versions of the Article. 